\title{
Genetically engineered materials: Proteins and beyond
}

\author{
Jingjing $\mathrm{Wei}^{1 \dagger}$, Lianjie $\mathrm{Xu}^{2 \dagger}$, Wen-Hao $\mathrm{Wu}^{2}$, Fei Sun ${ }^{3^{*}}$ \& Wen-Bin $\mathrm{Zhang}^{2 *}$ \\ ${ }^{1}$ College of Chemical and Environmental Engineering, Anyang Institute of Technology, Anyang 455000, China; \\ ${ }^{2}$ Beijing National Laboratory for Molecular Sciences, Key Laboratory of Polymer Chemistry \& Physics of Ministry of Education, Center for \\ Soft Matter Science and Engineering, College of Chemistry and Molecular Engineering, Peking University, Beijing 100871, China; \\ ${ }^{3}$ Department of Chemical and Biological Engineering, The Hong Kong University of Science and Technology, Clear Water Bay, Kowloon, \\ Hong Kong SAR, China
}

Received October 25, 2021; accepted December 21, 2021; published online January 26, 2022

\begin{abstract}
Information-rich molecules provide opportunities for evolution. Genetically engineered materials are superior in that their properties are coded within genetic sequences and could be fine-tuned. In this review, we elaborate the concept of genetically engineered materials (GEMs) using examples ranging from engineered protein materials to engineered living materials. Proteinbased materials are the materials of choice by nature. Recent progress in protein engineering has led to opportunities to tune their sequences for optimal material performance. Proteins also play a central role in living materials where they act in concert with other biological components as well as nonbiological cofactors, giving rise to living features. While the existing GEMs are often limited to those constructed by building blocks of biological origin, being genetically engineerable does not preclude nonbiologic or synthetic materials, the latter of which have yet to be fully explored.
\end{abstract}

protein, genetic engineering, self-assembly, living materials

Citation: Wei J, Xu L, Wu WH, Sun F, Zhang WB. Genetically engineered materials: Proteins and beyond. Sci China Chem, 2022, 65: 486-496, https://doi.org/ 10.1007/s11426-021-1183-x

\section{Introduction}

The Materials Genome Initiative (MGI) was proposed in 2011 with the ultimate goal to revolutionize the conventional paradigm of materials development continuum, especially to reduce the time span of materials discovery, design and deployment [1]. As being pointed out in the MGI White Paper, the meaning of genome within non-biological contexts is "a fundamental building block toward a larger purpose". Thus, to make a material genetically-encodable, a comprehensive understanding of the relationships between the properties in all aspects and the arrangement of atoms within the material is necessary [2]. To address this fundamental problem, a combinatorial and systematic methodology integrating

$†$ These authors contributed equally to this work.

*Corresponding authors (email: wenbin@pku.edu.cn; kefsun@ust.hk) computational, experimental, and digital tools is proposed. Although remarkable progress has been achieved in the context of MGI, it remains challenging to realize genetic encodability in most of the existing synthetic materials [3]. On the other hand, however, nature has provided valuable and diverse prototypes of genetically-engineered materials in living systems and some of them can now be artificially engineered and repurposed at the genetic level towards smart and even living materials $[4,5]$.

The diversity and plasticity of living systems are the outcomes of evolution. It was said that nothing makes sense in biology except in the light of evolution. Evolution occurs when information (genotype) and function (phenotype) close the loop where replication with errors generates diversity and selection enriches the desired progeny. While evolution is typically associated with living systems, this algorithm still holds at the molecular level, as demonstrated by the in vitro 
evolution techniques such as systematic evolution of ligands by exponential enrichment and directed evolution via ribosomal display [6,7]. The Central Dogma of Biology holds that information flows from DNA to RNA and further to proteins. Proteins, the workhorse of life, also constitute the materials of choice in living systems, which have taught us a lot about material design and engineering, as shown in silks, hairs, keratin, as well as many other molecular materials like enzymes. The concept of genetically engineered materials (GEMs) has emerged in recent years to describe, in most cases, those man-made materials comprising artificial peptides or recombinant proteins with properties engineered for a specific purpose [8].

Protein-based, genetically engineered materials can be recognized at three hierarchies: molecules, assemblies, and systems. Broadly speaking, discrete folded proteins can be considered as a simple form of GEMs. Not only are they responsible for functions such as enzymatic catalysis, signal transduction, molecular transport, and so on, but also serve to construct materials at higher hierarchy. Folded proteins could further assemble into aggregates or ordered assemblies, be it discrete or infinite. Forming assemblies not only increases the protein stability, but may also add to the complexity of protein architectures, giving rise to new functions, such as structural support and functional regulation [9]. The assembly may also lead to bulk materials like silk. Artificial protein assemblies have been common in protein materials and may take a variety of forms ranging from zero-dimensional (0D) discrete nanocages, 1D fibers, 2D layers, to 3D micelles, phase-separated microstructures, ordered frameworks and crystals, and further to macroscopic gels [10-15]. The properties and functions of these entirely protein-based assemblies are encoded on the genes of the corresponding protein precursors and thus are fully programmable, or even evolvable. An even more complex system may result from an interacting network of biomolecules [16], as seen in the complex and diverse gene circuits and biological pathways found in living organisms. With the development of synthetic biology, it is possible to mimic this systems' level of control to create engineered living materials, or materials with living features. Such materials are considered as genetically engineered materials at the systems level [17]. In this highlight review, we discuss the concept of genetically engineered materials at these three different levels from molecules, to assemblies, and further to systems with increasing size and complexity (Figure 1). A brief summary of these materials are also provided in Table 1.

\section{Genetically engineered protein building blocks}

Protein molecules represent a class of prototypical GEMs,

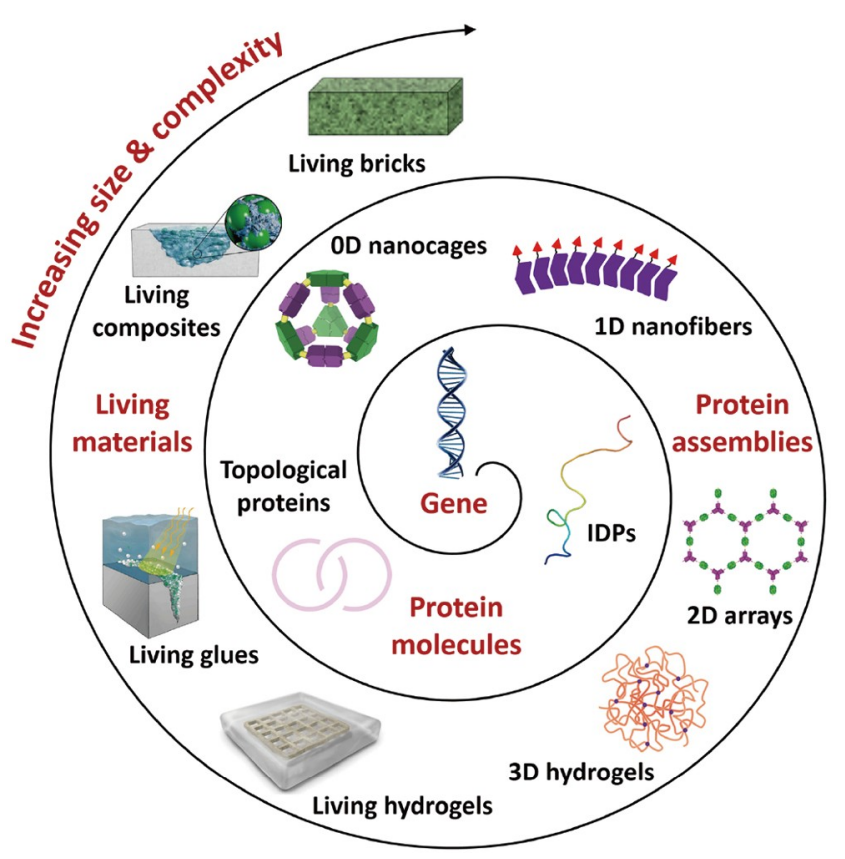

Figure 1 Overview of genetically engineered materials. Genetically engineered materials are rooted in their genes. The information flows from genes to RNA and further to proteins by translation. The properties and functions emerge across multiple scales from single-molecule level to assemblies' level and further to systems' level with increasing size and complexity (color online).

which can also serve as essential building blocks to create higher-order GEMs. The vast protein sequence space has provided numerous opportunities for expanding the repertoire of these building blocks. Among them, natural proteins constitute an abundant source of functional motifs and have currently inspired the design of many GEMs, but our pursuit of advanced protein-based biomaterials, enabled by powerful protein engineering tools, has already gone beyond the paradigm rigidly set by nature, thus leading to a much expanded structural and functional scope. To develop functional proteins, the essential units of higher-order GEMs, there are mainly two approaches: (1) engineering existing proteins toward a specific purpose; (2) finding new meaningful sequences either by gene mining from the plethora of functional sequences in nature or by de novo design of artificial functional sequences.

Although meaningful proteins are extremely rare within the vast, incomprehensively large sequence space, billions of years of evolution has inexorably swept away the debris and left behind a plethora of selected protein families in the living world. Fortunately, these folded structures are more conserved than their sequences and functions, making it possible to derive new functional sequences from the same scaffold by means of site-specific mutagenesis, domain insertion/fusion/deletion, domain swapping, circular permutation, split-and-reconstitution, and so on. Hence, new protein building blocks can be conveniently developed from existing 
Table 1 Summary of exemplary genetically engineered materials (GEMs)

\begin{tabular}{|c|c|c|}
\hline GEMs & & Examples \& applications \\
\hline \multirow{2}{*}{ Protein molecules } & Building blocks & $\begin{array}{c}\text { Intrinsically disordered proteins, star proteins, protein knots, lasso proteins, protein rotaxanes, } \\
\text { protein [n]catenanes, etc. }\end{array}$ \\
\hline & Functions & Reactive pairs, industrial enzymes, antibodies, protein therapeutics, etc. \\
\hline \multirow{4}{*}{ Protein assemblies } & $0 \mathrm{D}$ & $\begin{array}{l}\text { Nanocages like viral capsids, ferritins, encapsulin, chaperonins as nanocarriers, nanoreactors, } \\
\text { drug delivery vehicles, synthetic vaccines, etc. }\end{array}$ \\
\hline & $1 \mathrm{D}$ & Silk, curli fiber, amyloid etc., as structural components \\
\hline & $2 \mathrm{D}$ & S-layer as protective armor \\
\hline & $3 \mathrm{D}$ & Micelles, phase-separated microstructures, frameworks, crystals, hydrogels \\
\hline Living materials & $\begin{array}{l}\text { Escherichia coli } \\
\text { signals and giving }\end{array}$ & $\begin{array}{l}\text { biofilms integrated with gold nanoparticles; encapsulated } E \text {. coli hybrids capable of sensing mol } \\
\text { cence output; E. coli biofilms with light-guided gradient mineralization; Bacillus subtilis biofilm } \\
\text { can be 3D printed and regenerated }\end{array}$ \\
\hline
\end{tabular}

ones to fit into our demands. Even new functional modes could arise. The recent introduction of "covalent" proteinprotein interactions is a prominent example. Conventionally, most of the natural protein-protein interactions are physical in nature with limited affinity. A protein-protein interaction involving covalent bonding possesses an infinite affinity by definition and is thus fundamentally different. In 2010, Howarth et al. [18] developed a peptide-protein reaction pair that can reconstitute and form an isopeptide bond, albeit with a moderate efficiency, by dissecting the major pilin protein Spy0128 from Streptococcus pyogenes. In 2012, the same group [19] reported the development of SpyTag/SpyCatcher chemistry, a highly efficient isopeptide-forming peptideprotein pair. Regarded as a prototype of genetically encoded "click" chemistry, it has already gained traction with a broad biotechnology community [20]. The combined use of directed evolution and rational design led to further optimized pairs, approaching a quantitative yield and an instantaneous reaction rate [21,22]. The strategy for developing these chemistries appears to be generalizable; new reactive pairs can be developed in a similar fashion by splitting and engineering other protein homologs harboring isopeptide or ester bonds [23]. Together, these studies have established covalent bonding as a new mode of protein-protein interaction; the molecular recognition between the peptide and the protein ensures the specificity, while the subsequent autocatalysis warrants the yield. The short SpyTag also serves as a bridge for linking proteins with other synthetic molecules [24]. Since these reactants are entirely made of natural amino acids, they are fully genetically encodable, which is invaluable for performing these chemistries in biological systems. As a side-chain ligation tool, they also render a unique opportunity to synthesize nonlinear proteins.

The advent of "assembly-reaction" synergy in supramolecular chemistry has enabled the synthesis of numerous exotic molecules with unusual chemical topologies. Translating this concept into the macromolecular context, Zhang and co-workers [25] used an entangling protein motif in conjunction with genetically encoded click chemistry [20] to achieve the selective synthesis of proteins with complex topology. A series of topological proteins have been synthesized in cellulo. Examples include the passive template synthesis of protein [n]catenanes [25-27] and lasso proteins [28] and the active template synthesis of protein heterocatenanes [29] (Figure 2a). Functional improvements have been identified for these unconventional topologies, including enhanced stability against proteolytic degradation and thermal/chemical/mechanical denaturation (Figure 2b) [30,31]. Coincidently, similar effects have been observed in a few rare examples in nature. For example, citric synthase from the thermophile Pyrobaculum aerophilum is a naturally occurring protein catenane and exhibits a melting temperature $\left(T_{\mathrm{m}}\right) 10.5^{\circ} \mathrm{C}$, higher than that of the open-ended dimer [32]. More impressively, the bacteriophage HK97 capsid, which is a topologically linked chainmail with remarkable stability, partially resists denaturation at $5 \mathrm{M} \mathrm{GuHCl}[33,34]$. Topology has thus been added into protein engineering as a new dimension beyond the two conventionally used parameters, length and sequence [35,36]. This new parameter converts a protein molecule from a linear, one-dimensional polypeptide chain into a multi-chain, multi-dimensional species. These artificial topological proteins, with their versatile designability and improved stability and dynamics, will greatly expand the repertoire of the building blocks needed for the construction of higher-order GEMs.

While engineering existing proteins, either within the linear paradigm or to make their topological variants, provides rich opportunities to tailor their structure and function, finding entirely new functional sequences is of even more interest. One can use gene mining to discover the beautiful sequences already present in the biological system. But, even the evolution of life on earth has tested only a small fraction of the protein sequence space. Hence, de novo design of proteins open the avenue to a much expanded chemical space of genetically engineered materials. Recently, considerable progress has been made in the area of de novo protein design 
(a)
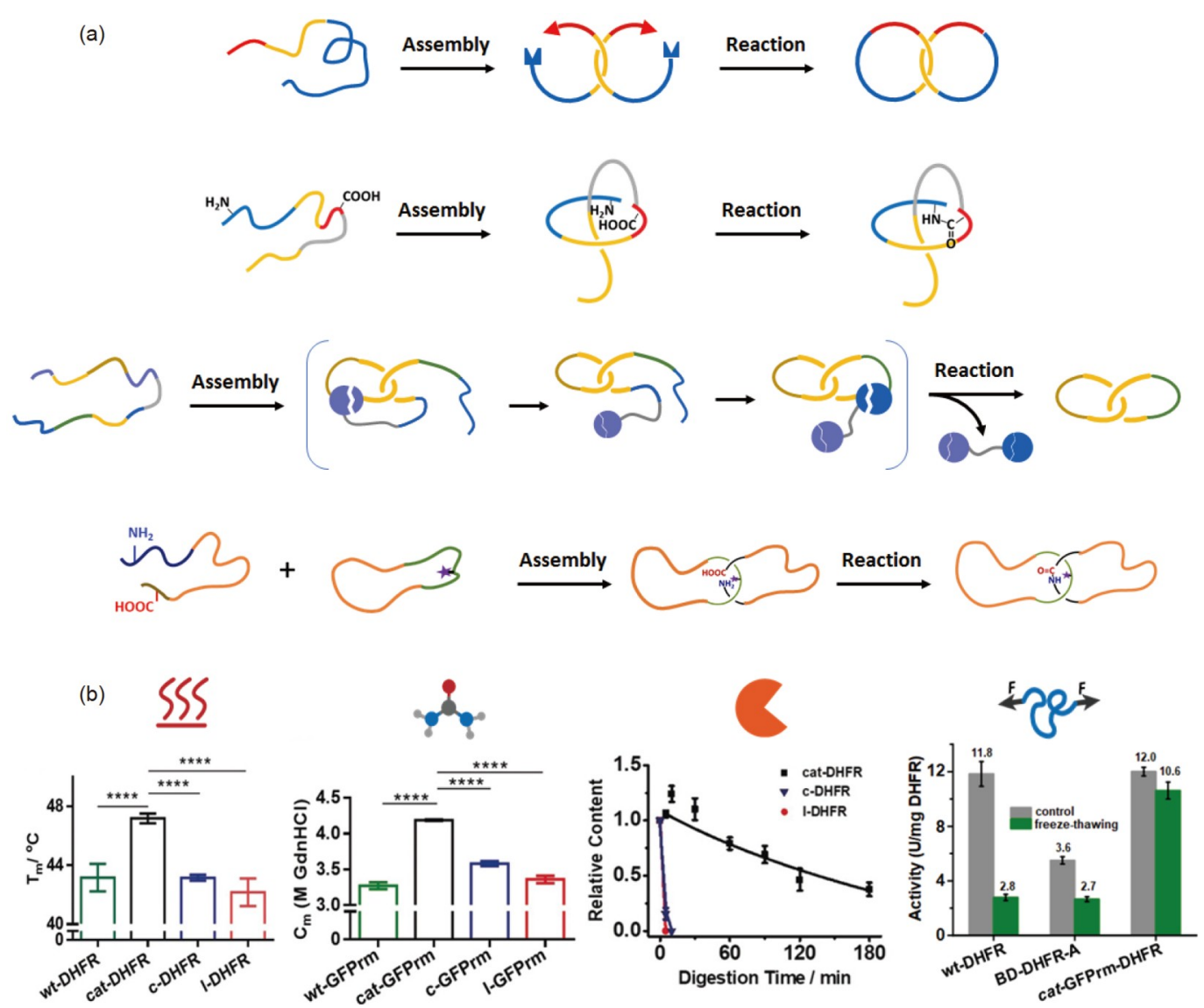

Figure 2 Proteins with unconventional topology. (a) The assembly-reaction synergy for cellular synthesis of topological proteins: protein homocatenane synthesis from intermolecular entwining (top) (adapted with permission from Ref. [25], copyright 2016 John Wiley \& Sons), lasso protein synthesis from intramolecular entwining (second) (adapted with permission from Ref. [28], copyright 2020 John Wiley \& Sons), protein heterocatenane synthesis from intramolecular entwining followed by split-intein mediated ligation (third) (adapted with permission from Ref. [26], copyright 2020 John Wiley \& Sons), and protein heterocatenane synthesis by the active template (adapted with permission from Ref. [29], copyright 2019 John Wiley \& Sons). (b) Functional benefits imparted by chemical topology of proteins include enhancement stability against thermal denaturation (left), chemical unfolding (middle left), proteolytic digestion (middle right), and freeze-and-thaw induced mechanical unfolding (right) (adapted with permission from Refs. [29,30], copyright 2019 John Wiley \& Sons) (color online).

with the aid of computation, data science and machine learning [37]. It has become possible to design a protein, from small to medium in size, with a reasonable rate of success. For example, using the Rosetta method, Baker et al. [38-43] have designed $0 \mathrm{D}$ protein nanocages, 1D fibers, 2D lattices, artificial enzymes, GFP analogs, interleukin analogs and so on. The recent triumph of AlphaFold in highly accurate protein structure prediction has been considered as revolutionary $[44,45]$. Since structure prediction and protein design are the two sides of the same problem, we anticipate the advent of a similar game changer on the inverse folding problem in the near future [46]. While structured protein molecules have been the current focus of de novo design, there remains a lot to learn from basic polymer physics and naturally occurring protein sequences, especially those without orderly 3D structures but with well-defined functions. One should keep in mind that functional proteins have a broad continuum in the degree of order, from completely disordered to highly structured. Strengthened abilities to de novo design these molecules, be it ordered or disordered, will open the whole sequence space to genetically engineered materials.

\section{Genetically engineered protein assemblies}

Materials are usually homogenous or heterogeneous assemblies of molecules that could collectively accomplish certain functions. Proteins are extremely rich in their mutual interactions or interactions with other types of molecular components. Thus, protein molecules by themselves can fold into various shapes and structures, which can further assemble into complex structures across different length scales and generate collective and cooperative functions. Such protein assemblies are also considered as genetically engineered protein materials.

One of the most prevalent protein assemblies are protein nanocages, which can be viewed as a type of OD material. While proteins are often used as the bioactive component in self-assembled nanoparticles in biomedicine [47], naturally 
existing protein nanocages, such as viral capsids, ferritins, encapsulin and chaperonins, can also serve as carriers of functional molecules (e.g., DNA, RNA, proteins, or metal ions) and/or nanoreactors for chemical reactions (e.g., riboflavin biosynthesis) [48]. Repurposing these natural protein cages for biomaterials has been nicely demonstrated for various biomedical applications, such as drug delivery vehicles, synthetic vaccines, and catalytic nanoreactors [49]. Moreover, recent success in the computational design of both one- and multi-component protein cages has opened the gate to diverse 0D protein materials [38-41]. For example, antigens like SARS-CoV-2 receptor-binding domains (RBD) have been fused onto a computationally designed protein cage component I53-50A and co-assembled with I53-50B into a nanoparticle vaccine displaying $60 \mathrm{RBD}$ on surface and elicit potent and protective antibody responses against SARS-CoV-2 in mice (Figure 3a) [50]. These designer protein cages, which are genetically encoded, highly stable and robustly assembled, have been used as a novel platform for developing "plug-and-display" vaccines, in which reactive tags tethered on their surfaces allow for the decoration with heterologous antigens [50,51]. One should note that such protein cages can be evolved for even further improved stability, encapsulation capacity and substrate specificity, offering opportunities for future development $[52,53]$. In addition, various protein assemblies like proteasomes and molecular chaperones are also considered 0D owing to their discrete nature and small size.

Proteins can also assemble into fiber-like 1D materials, some of which, with mass production and wide use, have generated long-lasting impacts on human society; about five millennia ago, China's civilization learned to raise Bombyx mori (silkworms) and to weave their delicate fibers into fine silk cloth, as well as substitute materials of paper for writing and recording. Empowered by modern genome engineering tools such as TALEN and CRISPR-Cas9, mankind now is able to genetically engineer the silkworms to produce silk materials with unprecedented properties, including those amenable to further covalent decoration by functional proteins both in vivo and in vitro [54-56]. Spider silk, another category of naturally occurring 1D protein material, is gaining traction with materials scientists in recent years for its exceptional mechanical and biochemical properties; dragline silk rivals steel in strength and is said to be nonimmunogenic and biocompatible for biomedical applications [57]. Unlike silkworms, spiders are territorial and cannibalistic and thus cannot be farmed, which prevents the mass production of spider silk for common use. Although modern recombinant DNA technology has enabled the production of genetically engineered spider silk using bacterial cells, with one case even able to recapitulate those naturally occurring ones in mechanics [58], the mass production of these recombinant protein materials has yet to be realized. In addi- tion to silk, other types of $1 \mathrm{D}$ protein materials are also bountiful in nature. As essential components of biofilms, the protein fibers impart considerable mechanical stability to the biofilms and also display multiple biological signals [59]. Representative curli fiber monomers include Escherichia coli CsgA and Bacillus subtilis TasA. Microorganisms often exploit amyloids, though considered detrimental in human bodies, to gain a competitive edge over their competitors or enemies [60]. These amyloid motifs are also building blocks of choice for constructing genetically engineered materials. For example, Collier and co-workers [61] fused $\beta$-sheet fibrillizing domains to different fluorescent proteins, of which the co-assembly was induced upon the addition of $\beta$-sheet fibrillizing peptides, thus leading to the formation of nanofiber materials displaying a controllable combination of proteins (Figure 3b).

As another low-dimensional material, 2D protein assemblies are not uncommon either. An excellent example is the naturally occurring bacterial S-layer assembled on the cell surface which comprises of an orderly monomolecular layer of identical proteins known as S-layer proteins. This natural protein material mainly serves as an armor to protect bacterial cells, while also being engaged in many other functions like enzyme display, cellular communication, and pathogenesis [62]. Recombinant S-layer proteins fused with various domains can be produced heterologously and the resulting products can robustly self-assemble into ordered $2 \mathrm{D}$ arrays in vitro in spite of the additional motifs attached. Thanks to their robustness, genetic encodability and high density of functional groups, S-layers have served as a versatile platform for designing diverse genetically engineered 2D materials, which enabled functional display of enzymes, ligands, antigens, antibodies, inorganic nanoparticles, and so forth $[63,64]$. Lanthanide-binding peptide tags were fused to the S-layer protein of Caulobacter crescentus, leading to the creation of engineered $2 \mathrm{D}$ protein films with high absorption capacity toward rare earth elements [65]. Decoration of the S-layer protein with streptavidin, which enables further immobilization of biotinylated molecules, has given rise to a general platform for creating various functional materials [66]. Alternatively, incorporating peptide sequences like SpyTag or SnoopTag into the S-layer proteins has allowed covalent, yet robust and flexible functionalization of these 2D platforms [67]. Computational design of 2D protein lattices has also achieved considerable progress, making it possible to prepare $2 \mathrm{D}$ protein lattices with various symmetry from one or binary protein components. The design tolerates the reconfiguration of symmetry, composition, and surface features, which can be leveraged to modulate cell responses (Figure 3c) [68].

Protein materials can also take the form of macroscopic 3D protein assemblies with well-defined or amorphous network structures. To distinguish them from the aforementioned 0D 
Building blocks

(a)

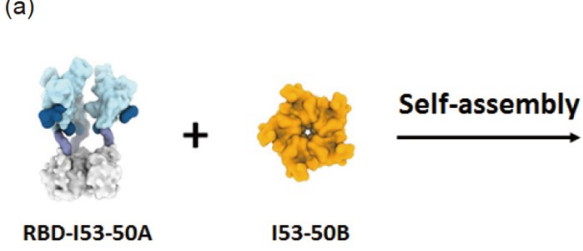

\section{Protein materials}

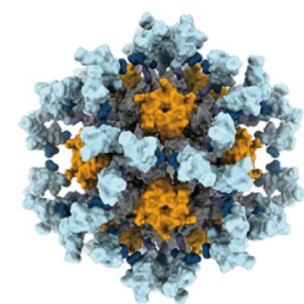

SARS-CoV-2 RBD-153-50 nanoparticle

(b)
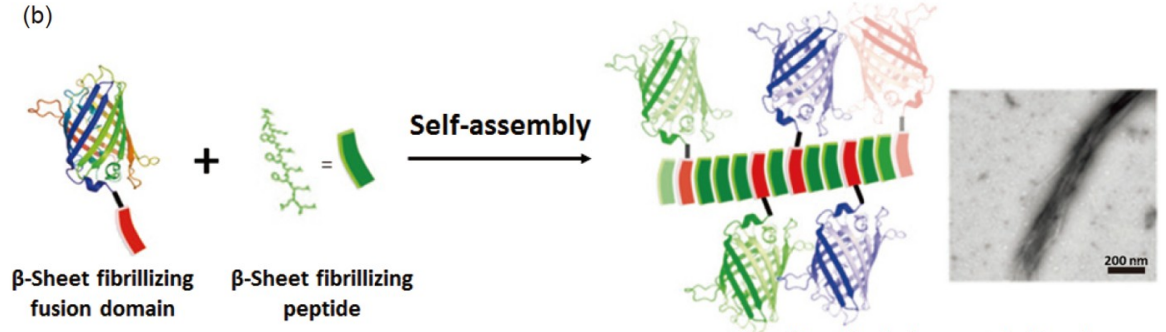

(c)
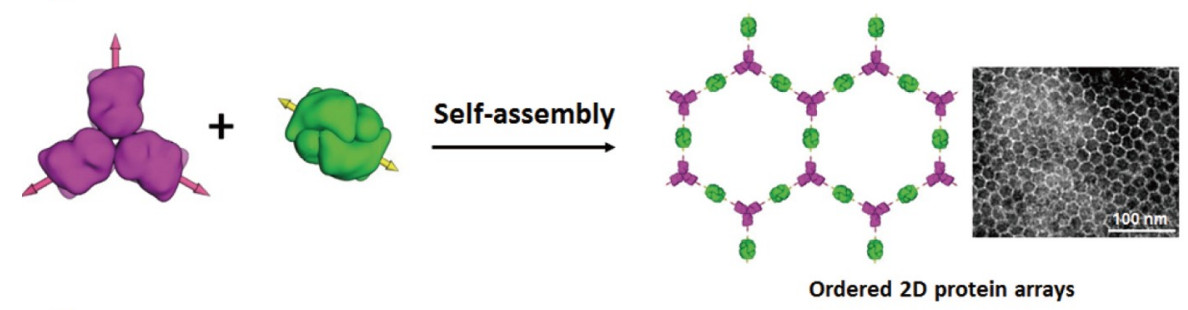

(d)

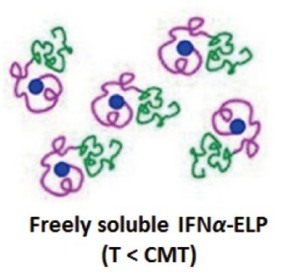

Thermally induced self-assembly
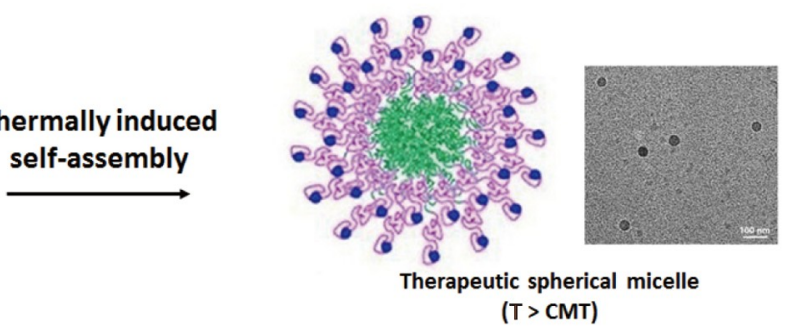

Figure 3 Genetically engineered protein assemblies. (a) 0D protein nanocages such as designed protein nanocages displaying the receptor binding domain (RBD) of SARS-CoV-2 [50]; (b) 1D protein nanofibers assembled from $\beta$-sheet fibrillizing peptides (adapted with permission from Ref. [61], copyright 2021 Springer Nature); (c) 2D protein arrays assembled by oligomeric proteins with designed interface (adapted with permission from Ref. [68], copyright 2014 Nature Publishing Group); (d) 3D protein micelles by thermally induced self-assembly of therapeutic proteins fused with ELP (T: temperature; CMT: critical micelle temperature; adapted with permission from Ref. [73], copyright 2020 Elsevier) (color online).

assemblies, we can make the distinction mainly based on their size; the $0 \mathrm{D}$ assemblies are nanoscale, with precisely defined atomic arrangement, while the 3D assemblies are much larger, typically at meso- to macroscale (larger than micrometer). In the context of supramolecular chemistry, micelles and vesicles are less ordered 3D assemblies, and frameworks and crystals are much more ordered. Amphiphilicity of surfactants is often the driving force behind these 3D assemblies. Some natural proteins, albeit rare, can act like surfactants under physiological conditions [69]. For example, the túngara frog uses a surfactant protein called Ranaspumin-2 to create foam nests, thus providing protective and biocompatible shelters for eggs and embryos [70]. Artificial protein-based surfactants have also been designed by connecting a hydrophobic polypeptide with a hydrophilic one. Champion and co-workers [71] used oppositely charged leucine-zippers to induce the assembly of two recombinant proteins, hydrophilic GFP and hydrophobic elastin-like polypeptide (ELP), into supramolecular protein amphiphiles, which, upon heating, further assembled into vesicles with the potential for controlled drug delivery. Gao and co-workers $[72,73]$ designed an artificial amphiphilic protein by genetically fusing an ELP with a hydrophilic protein drug, interferon- $\alpha$ (IFN $\alpha$ ). The IFN-ELP diblock copolypeptide assembled into a spherical micelle, $48 \mathrm{~nm}$ in diameter, with an ELP core and an IFN shell (Figure 3d). Not only does the large size of the assembly prohibit kidney filtration and extend the lifetime of the protein drug in vivo, the micelle also 
exhibits a multivalent effect with enhanced stability and drug potency.

Protein phase separation has received great attention from the research community in recent years, for its newfound role in biological regulation. In nature, the protein phase separation underlies the formation of larger 3D protein assemblies, such as the droplets or granules inside cells, by which membraneless organelles emerge and regulate cellular processes [74]. For example, $\mathrm{P}$ granules segregate with the $\mathrm{P}$ lineage during embryogenesis and help maintain germ cell integrity. Protein phase separation also plays key roles in regulating synapses and accounts for the formation of semimembrane-bound postsynaptic density [75]. The protein phase separation is also believed to be a mechanism by which cells exert spatiotemporal control over the expression of functional proteins in response to external signals. The protein phase separation process is often driven by multivalent interactions among either intrinsically disordered or folded globular proteins, which may be modulated by posttranslational modifications (e.g., phosphorylation and methylation) in response to local environmental variations in stress, temperature and so on.

To recapitulate the delicate phase separation in artificial materials, one can rationally design protein building blocks with tailored physical properties based on very simple rules. ELPs, consisting of VPGXG repeats (where $\mathrm{X}$ is the guest residue), are a class of artificial intrinsically disordered proteins inspired by natural tropoelastin [76]. The correspondence between the thermo-responsive phase transition behavior and the chemical composition of ELPs has been well established [77]. Chilkoti and co-workers [78] have systematically studied in microdrops the phase separation of a group of ELPs with varied guest residue, chain length and block architecture, leading to a set of rules that govern the ELP-ELP interactions and the resulting phase-separation. Recently, the same group created complex self-assembled microarchitectures comprising the mixtures of artificial ELPs and partially ordered proteins across multiple length scales simply using droplet microfluidics and stepwise heating, which has opened the door to a variety of genetically programmable structures (Figure 4a) [79]. These researchers further engineered the intrinsically disordered proteins (IDPs) based on the sequence-property relationship, thus enabling the modulation of phase separation in living cells [80]. Conticello and co-workers [81] created pH-dependent interconversion of collagen tubes and sheets using heteromeric pairs of collagen-mimetic peptides with carefully selected number and position of charged residues for charge complementary. The controllable transformation between different assembly structures and morphologies would be promising in designing versatile and smart carriers of a diverse spectrum of molecular payloads.

In addition to phase separation, stable physical/chemical crosslinking provides an alternative approach for transforming protein building blocks into macroscopic genetically engineered materials like hydrogels. Crosslinking proteins into a gel allows one to take advantage of their ecological diversity and functional versatility. Crosslinking ELPs typically gives rise to elastic hydrogel materials, which in turn can serve as artificial extracellular matrices. Using the thiol-ene reaction, Murphy et al. [82] incorporated calmodulin in the polyethylene glycol (PEG) hydrogel, which demonstrated reversible volume transition in response to the cognate ligands of calmodulin. Cao et al. [83] have recently created a series of PEG-protein hydrogels through deliberate use of proteinprotein interactions, revealing a good correlation between the stability of the hydrogels and the mechanics at the singlemolecule level. However, these materials can only be loosely regarded as genetically engineered, as they often contain abiotic components like PEG, even though their biological activities are largely determined by the protein building blocks. Besides, the crosslinking chemistry needs to be carefully chosen so as not to interfere with the folded structure of proteins and compromise their activity.

To circumvent this limitation, it is highly desirable to have genetically engineered materials, including their crosslinkers within, which are entirely composed of protein molecules. In 1998, Tirrell and co-workers [84] were among the first to demonstrate the creation of entirely engineered proteinbased hydrogels comprising physical networks of self-assembling triblock copolypeptides. Owing to the sequencedependent, reversible oligomerization of the terminal leucine-zipper coiled coils, these materials were genetically programmable in terms of their macroscopic properties, particularly their responsiveness toward environmental cues such as $\mathrm{pH}$ and temperature. Sun and co-workers [85] reported the synthesis of a covalently crosslinked, entirely protein-based hydrogel, the first of its kind, using the aforementioned SpyTag-SpyCatcher chemistry (Figure 4b). "The network of spies" or "Spy network" was coined to describe this hydrogel. Since the gel is fully genetically encoded, interesting functions commonly associated with complex biomacromolecules but rare to conventional synthetic materials may be facilely introduced and faithfully preserved throughout the material synthesis. Thanks to the diversity of natural and designed proteins, various stimuliresponsive and functional smart hydrogels have been created using different protein building blocks, such as photoresponsive hydrogels comprising the $\mathrm{B}_{12}$-dependent photoreceptor $\mathrm{CarH}_{\mathrm{C}}$ protein and capable of controlled release of proteins and living cells (Figure 4c), metalloprotein-containing hydrogels for selective sequestration of heavy metals (e.g., oceanic uranium, and chromate), as well as hydrogels containing antimicrobial proteins [86-89]. One drawback of these genetically engineered hydrogels lies in their weak mechanics, which can be attributed to the large size of the 


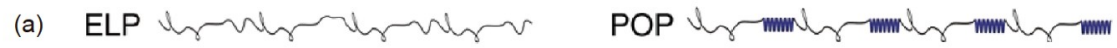
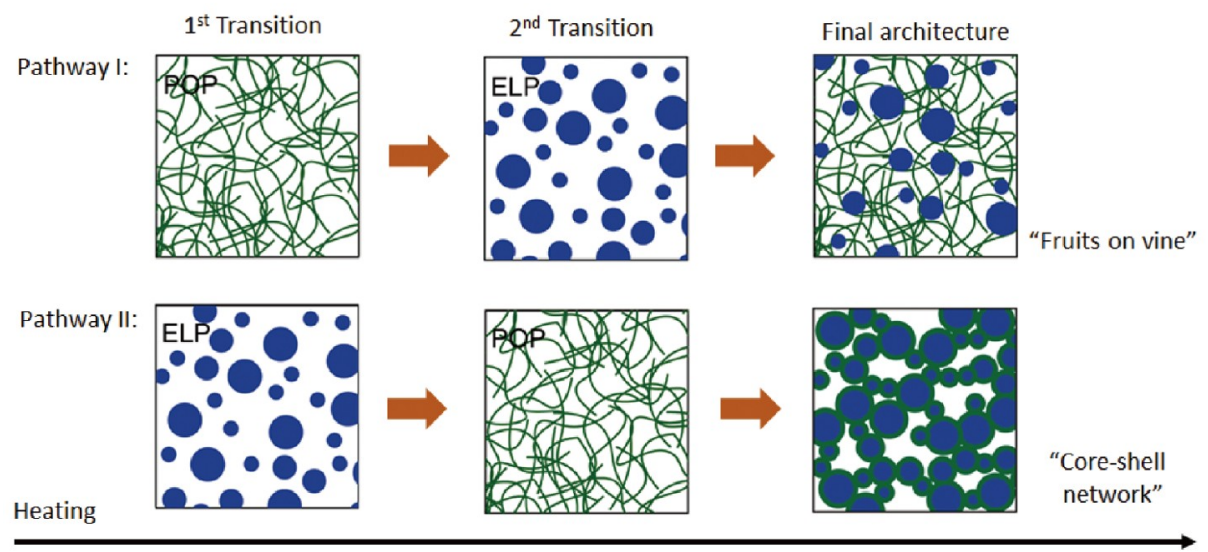

(b)

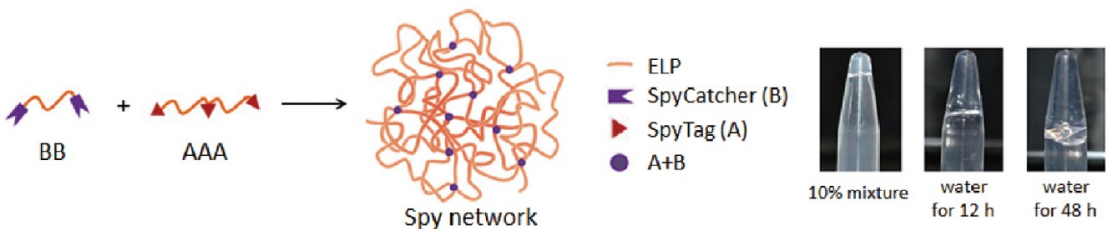

(c)

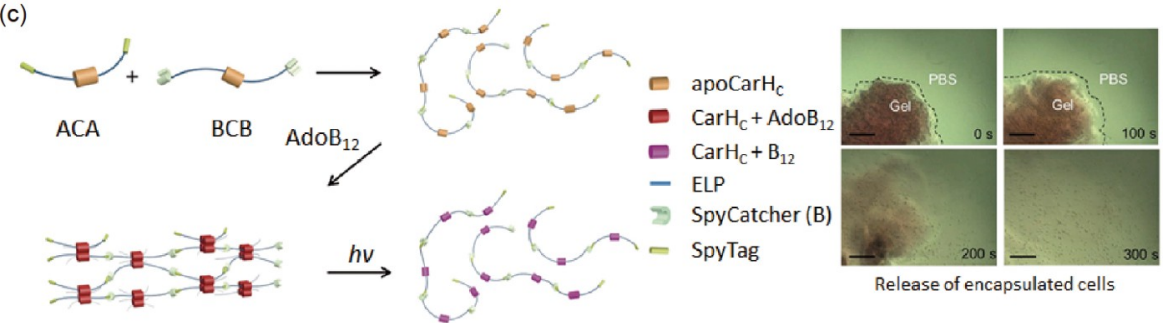

Figure 4 Genetically engineered protein materials. (a) Formation of complex microparticle architecture from responsive intrinsically disordered proteins (adapted with permission from Ref. [79], copyright 2020 Springer Nature); (b) covalent protein-based hydrogel using SpyTag-SpyCatcher chemistry (adapted with permission from Ref. [85], copyright 2014 National Academy of Sciences); (c) smart hydrogel for controlled release of living cells by incorporation of chemical- and light-responsive protein motifs (adapted with permission from Ref. [86], copyright 2017 National Academy of Sciences) (color online).

SpyTag/SpyCatcher crosslinker and correspondingly the low crosslinking density. As to conventional synthetic hydrogels, a variety of strategies have been developed to enhance their mechanical stability, including the creation of those notable network structures, nanocomposite network, covalent double network, chemical-physical double network, slide-ring network, and ionically crosslinked network [90]. We envision that similar strategies can be adopted to improve the mechanics of these entirely protein-based materials.

\section{Genetically engineered living materials}

The ever-growing challenges mankind nowadays faces, from health, energy to environment, have necessitated a paradigm shift in materials science and engineering; our design parameters and consideration cannot solely eye on the molecular components. Rather, what we need is a shift from genetically encoded molecules to genetically encoded systems- reminiscent of a similar molecules-to-systems shift that occurred on this planet billions of years ago and provided the bedrock foundation for all later forms of life. From materials scientist's perspective, life on earth is a type of naturally occurring, genetically encoded living material that is consistently evolving, adapting to and/or reshaping surrounding environments; a living species is not merely a bag of organic molecules, but rather a system endowed with emergent properties by the assembly of genetically encoded molecules, alongside some abiotic ones. In this vein, synthetic biologists conceived the concept of engineered living materials. Through the integration of synthetic biology and materials science, several prototypes of engineered living materials have since been developed.

Engineered living materials are materials with living features, including self-organization, self-sustaining, adaptability, reproducibility, and evolvability. One straightforward way to make engineered living materials is to incorporate living cells as a material component. Stark et al. [91] created 
a primitive engineered living materials by embedding $\mathrm{Pe}$ nicillium roqueforti cells between a polymer base layer and a nano-porous top layer, where the metabolism of these cells generated a self-cleaning living surface. This design principle was later adopted for converting penicillin-producing fungi into a living antibiotic-releasing surface [92]. Nevertheless, these materials have yet to exhibit any prominent emergent property - a property that does not arise from a single material component but from an interacting system. Recent progress has pointed to the feasibility of creating advanced engineered living materials with emergent properties. For example, Lu et al. [93] created switchable conductive living biofilms by integrating genetically engineered E. coli cells with gold nanoparticles (GNPs). The living cells, under the delicate control of inducible genetic circuits, were able to produce and display GNP-binding protein fibers for integration into a systems material. In another study, with the aim to design wearable living sensors, Zhao et al. [94] engineered a collection of $E$. coli strains that not only can be induced by various chemicals but also participate in intercellular communications; these cells, when co-encapsulated within robust hydrogel-elastomer hybrid matrices, act in synergy to result in a responsive GFP expression with fluorescent signal as the output.

Another remarkable feature of a living system is its agile adaptability. Engineered cells, with the aid of genetic cir- cuits, can sense and respond to complex environmental signals in a concerted manner, which in turn alters not only the behavior of individual cells, but also their intercellular connections and spatial arrangement. Hence, both biochemical and mechanical properties of a living material can be tuned with great agility. For example, Zhong et al. [95] developed spatially controllable biomimetic mineralization within engineered E. coli biofilms in a light-dependent manner, where gradient mineral densities and mechanical properties were achieved (Figure 5a). This work represents a significant advance in designing highly adaptable living materials. Moreover, engineered living materials have been designed to grow into a made-to-order shape. The same group engineered TasA protein, a major component of the biofilm extracellular matrix, into Bacillus subtilis to make it more hydrophilic [96]. The resulting gel-like biofilms became highly processible. Not only could they be printed into desirable 3D shapes, but also they could be regenerated on agar plates via simple imprinting (Figure $5 \mathrm{~b}$ ). These engineered living materials, with the combination of genetic programmability, unique material properties and living features, hold enormous prospects in that they can be delivered to remote areas where bulk transportation is difficult or even impossible, or applied under extreme conditions where they can self-grow into particular shapes and mature into functional forms.
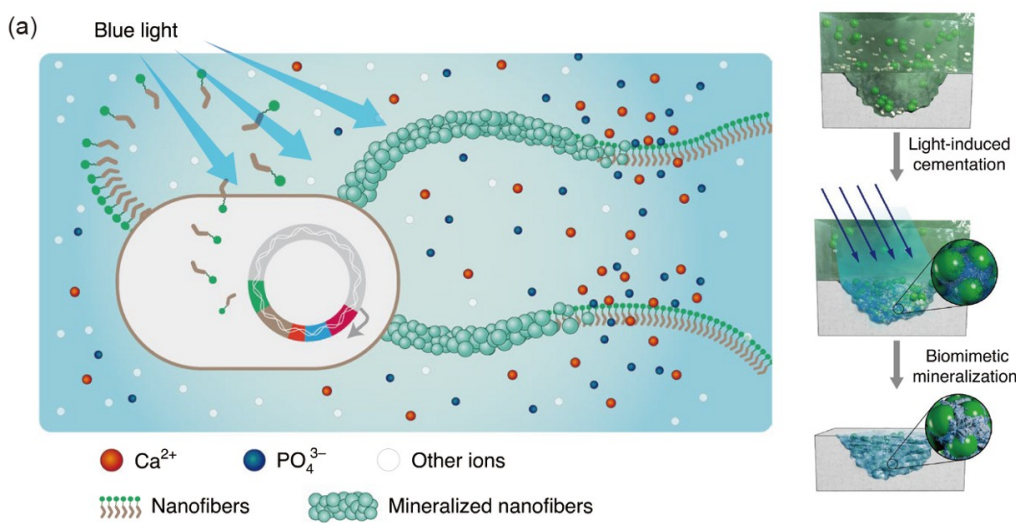

(b)
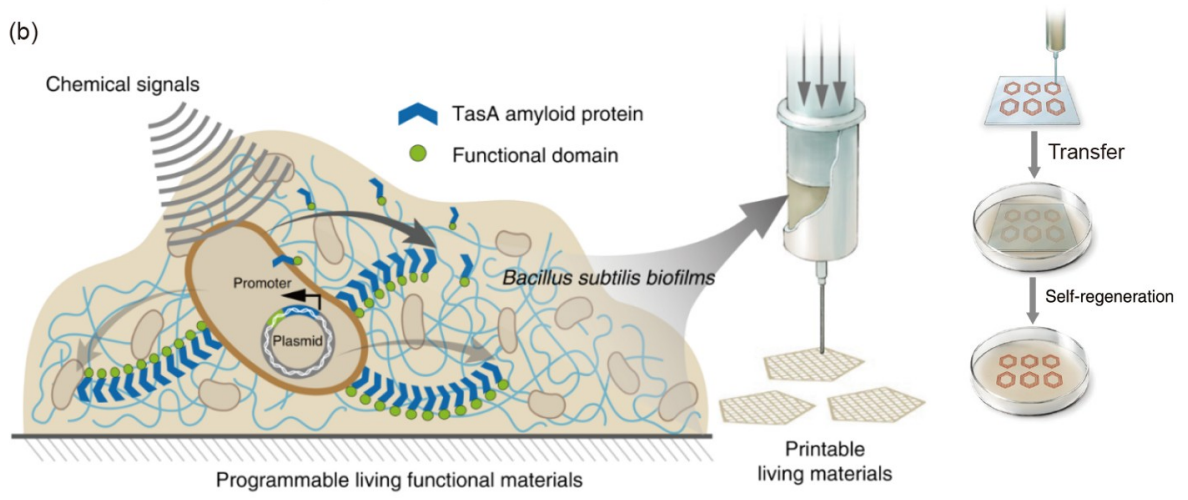

Figure 5 Genetically engineered living materials. (a) Light-inducible living composite materials using biomimetic mineralization (adapted with permission from Ref. [95], copyright 2020 Springer Nature); (b) printable and regeneratable living materials based on Bacillus Subtilis biofilms (adapted with permission from Ref. [96], copyright 2018 Springer Nature) (color online). 


\section{Summary}

In this highlight review, we discussed genetically engineered materials from common protein molecules, to their functional assemblies, and further to the interacting systems as engineered living materials. The essential feature of these genetically engineered materials is their ability to carry biological information and translate the information into a specific function. This correlation between the information and the function makes it possible to fine-tune the properties and to evolve the material system, regardless of its length scale or detailed molecular mechanism. The recent development of engineered living materials has also ushered in a paradigm shift in basic materials science. With its integration with synthetic biology, genetically engineered materials will surely find real-world applications in the near future.

Acknowledgements This work was supported by the National Natural Science Foundation of China (21905001, 21925102, 21991132, 92056118), the National Key R\&D Program of China (2020YFA0908100), Beijing Municipal Natural Science Foundation (L182003), Beijing National Laboratory for Molecular Sciences (BNLMS-CXXM-202006), Guangdong Natural Science Foundation (GDST19EG22), the Science, Technology, and Innovation Commission of Shenzhen Municipality (JCYJ20190813094601656), and Clinical Medicine Plus X project of Peking University, Fundamental Research Funds for the Central Universities.

Conflict of interest The authors declare no conflict of interest.

1 de Pablo JJ, Jones B, Kovacs CL, Ozolins V, Ramirez AP. Curr Opin Solid State Mater Sci, 2014, 18: 99-117

2 Liu Z. Chin Sci Bull, 2014, 59: 1619-1623

3 de Pablo JJ, Jackson NE, Webb MA, Chen LQ, Moore JE, Morgan D, Jacobs R, Pollock T, Schlom DG, Toberer ES, Analytis J, Dabo I, DeLongchamp DM, Fiete GA, Grason GM, Hautier G, Mo Y, Rajan K, Reed EJ, Rodriguez E, Stevanovic V, Suntivich J, Thornton K, Zhao JC. npj Comput Mater, 2019, 5: 41-54

4 Shen L, Bao N, Zhou Z, Prevelige PE, Gupta A. J Mater Chem, 2011, 21: $18868-18876$

5 DiMarco RL, Heilshorn SC. Adv Mater, 2012, 24: 3923-3940

6 Gold L. J Mol Evol, 2015, 81: 140-143

7 Arnold FH. Angew Chem Int Ed, 2018, 57: 4143-4148

8 Tamerler C, Sarikaya M. ACS Nano, 2009, 3: 1606-1615

9 Zhang WB, Cheng SZD. Giant, 2020, 1: 100011

10 Sun H, Li Y, Yu S, Liu J. Front Bioeng Biotechnol, 2020, 8: 295-304

11 Kong G, Xiong M, Liu L, Hu L, Meng HM, Ke G, Zhang XB, Tan W. Chem Soc Rev, 2021, 50: 1846-1873

12 Zhu J, Avakyan N, Kakkis A, Hoffnagle AM, Han K, Li Y, Zhang Z, Choi TS, Na Y, Yu CJ, Tezcan FA. Chem Rev, 2021, 121: 1370113796

13 Luo Q, Hou C, Bai Y, Wang R, Liu J. Chem Rev, 2016, 116: 1357113632

14 Whyburn GP, Li Y, Huang Y. J Mater Chem, 2008, 18: 3755

15 Zhao L, Qiao S, Liu J. Sci China Chem, 2016, 59: 1531-1540

16 Xu L, Wang X, Sun F, Cao Y, Zhong C, Zhang WB. Curr Opin Solid State Mater Sci, 2021, 25: 100896

17 Nguyen PQ, Courchesne NMD, Duraj-Thatte A, Praveschotinunt P, Joshi NS. Adv Mater, 2018, 30: 1704847

18 Zakeri B, Howarth M. J Am Chem Soc, 2010, 132: 4526-4527

19 Zakeri B, Fierer JO, Celik E, Chittock EC, Schwarz-Linek U, Moy VT, Howarth M. Proc Natl Acad Sci USA, 2012, 109: E690-E697
20 Sun F, Zhang WB. Chin J Chem, 2020, 38: 894-896

21 Keeble AH, Banerjee A, Ferla MP, Reddington SC, Anuar INAK, Howarth M. Angew Chem Int Ed, 2017, 56: 16521-16525

22 Keeble AH, Turkki P, Stokes S, Khairil Anuar INA, Rahikainen R, Hytönen VP, Howarth M. Proc Natl Acad Sci USA, 2019, 116: 2652326533

23 Young PG, Yosaatmadja Y, Harris PWR, Leung IKH, Baker EN, Squire CJ. Chem Commun, 2017, 53: 1502-1505

24 Yin G, Wei J, Shao Y, Wu WH, Xu L, Zhang WB. Chin Chem Lett, 2021, 32: 353-356

25 Wang XW, Zhang WB. Angew Chem, 2016, 128: 3503-3507

26 Liu Y, Duan Z, Fang J, Zhang F, Xiao J, Zhang WB. Angew Chem Int Ed, 2020, 59: 16122-16127

27 Wu WH, Bai X, Shao Y, Yang C, Wei J, Wei W, Zhang WB. J Am Chem Soc, 2021, 143: 18029-18040

28 Liu Y, Wu WH, Hong S, Fang J, Zhang F, Liu GX, Seo J, Zhang WB. Angew Chem Int Ed, 2020, 59: 19153-19161

29 Da XD, Zhang WB. Angew Chem Int Ed, 2019, 58: 11097-11104

30 Wang XW, Zhang WB. Angew Chem, 2017, 129: 14173-14177

31 Wang XW, Zhang WB. Trends Biochem Sci, 2018, 43: 806-817

32 Boutz DR, Cascio D, Whitelegge J, Perry LJ, Yeates TO. J Mol Biol, 2007, 368: 1332-1344

33 Wikoff WR, Liljas L, Duda RL, Tsuruta H, Hendrix RW, Johnson JE. Science, 2000, 289: 2129-2133

34 Duda RL. Cell, 1998, 94: 55-60

35 Xu L, Zhang WB. Sci China Chem, 2017, 61: 3-16

36 Qu Z, Cheng SZD, Zhang WB. Trends Chem, 2021, 3: 402-415

37 Huang PS, Boyken SE, Baker D. Nature, 2016, 537: 320-327

38 King NP, Sheffler W, Sawaya MR, Vollmar BS, Sumida JP, André I, Gonen T, Yeates TO, Baker D. Science, 2012, 336: 1171-1174

39 King NP, Bale JB, Sheffler W, McNamara DE, Gonen S, Gonen T, Yeates TO, Baker D. Nature, 2014, 510: 103-108

40 Hsia Y, Bale JB, Gonen S, Shi D, Sheffler W, Fong KK, Nattermann U, Xu C, Huang PS, Ravichandran R, Yi S, Davis TN, Gonen T, King NP, Baker D. Nature, 2016, 535: 136-139

41 Bale JB, Gonen S, Liu Y, Sheffler W, Ellis D, Thomas C, Cascio D, Yeates TO, Gonen T, King NP, Baker D. Science, 2016, 353: 389-394

42 Dou J, Vorobieva AA, Sheffler W, Doyle LA, Park H, Bick MJ, Mao B, Foight GW, Lee MY, Gagnon LA, Carter L, Sankaran B, Ovchinnikov S, Marcos E, Huang PS, Vaughan JC, Stoddard BL, Baker D. Nature, 2018, 561: 485-491

43 Silva DA, Yu S, Ulge UY, Spangler JB, Jude KM, Labão-Almeida C, Ali LR, Quijano-Rubio A, Ruterbusch M, Leung I, Biary T, Crowley SJ, Marcos E, Walkey CD, Weitzner BD, Pardo-Avila F, Castellanos J, Carter L, Stewart L, Riddell SR, Pepper M, Bernardes GJL, Dougan M, Garcia KC, Baker D. Nature, 2019, 565: 186-191

44 Jumper J, Evans R, Pritzel A, Green T, Figurnov M, Ronneberger O, Tunyasuvunakool K, Bates R, Žídek A, Potapenko A, Bridgland A, Meyer C, Kohl SAA, Ballard AJ, Cowie A, Romera-Paredes B, Nikolov S, Jain R, Adler J, Back T, Petersen S, Reiman D, Clancy E, Zielinski M, Steinegger M, Pacholska M, Berghammer T, Bodenstein S, Silver D, Vinyals O, Senior AW, Kavukcuoglu K, Kohli P, Hassabis D. Nature, 2021, 596: 583-589

45 Tunyasuvunakool K, Adler J, Wu Z, Green T, Zielinski M, Žídek A, Bridgland A, Cowie A, Meyer C, Laydon A, Velankar S, Kleywegt GJ, Bateman A, Evans R, Pritzel A, Figurnov M, Ronneberger O, Bates R, Kohl SAA, Potapenko A, Ballard AJ, Romera-Paredes B, Nikolov S, Jain R, Clancy E, Reiman D, Petersen S, Senior AW, Kavukcuoglu K, Birney E, Kohli P, Jumper J, Hassabis D. Nature, 2021, 596: 590-596

46 Jendrusch M, Korbel JO, Sadiq SK. bioRxiv 2021, doi: 10.1101/ 2021.10.11.463937

47 Huang YK, Tian HR, Zhang MZ, He JL, Liu J, Ni PH. Chin J Polym Sci, 2021, 39: 1392-1402

48 Aumiller WM, Uchida M, Douglas T. Chem Soc Rev, 2018, 47: $3433-$ 3469

49 Zhang Y, Ardejani MS, Orner BP. Chem Asian J, 2016, 11: 2814 
2828

50 Walls AC, Fiala B, Schäfer A, Wrenn S, Pham MN, Murphy M, Tse LV, Shehata L, O'Connor MA, Chen C, Navarro MJ, Miranda MC, Pettie D, Ravichandran R, Kraft JC, Ogohara C, Palser A, Chalk S, Lee EC, Guerriero K, Kepl E, Chow CM, Sydeman C, Hodge EA, Brown B, Fuller JT, Dinnon Iii KH, Gralinski LE, Leist SR, Gully KL, Lewis TB, Guttman M, Chu HY, Lee KK, Fuller DH, Baric RS, Kellam P, Carter L, Pepper M, Sheahan TP, Veesler D, King NP. Cell, 2020, 183: 1367-1382

51 Marcandalli J, Fiala B, Ols S, Perotti M, de van der Schueren W, Snijder J, Hodge E, Benhaim M, Ravichandran R, Carter L, Sheffler W, Brunner L, Lawrenz M, Dubois P, Lanzavecchia A, Sallusto F, Lee KK, Veesler D, Correnti CE, Stewart LJ, Baker D, Loré K, Perez L, King NP. Cell, 2019, 176: 1420-1431

52 Wörsdörfer B, Woycechowsky KJ, Hilvert D. Science, 2011, 331: 589-592

53 Butterfield GL, Lajoie MJ, Gustafson HH, Sellers DL, Nattermann U, Ellis D, Bale JB, Ke S, Lenz GH, Yehdego A, Ravichandran R, Pun SH, King NP, Baker D. Nature, 2017, 552: 415-420

54 Minkner R, Xu J, Takemura K, Boonyakida J, Wätzig H, Park EY. $J$ Nanobiotechnol, 2020, 18: 159

$55 \mathrm{Xu}$ J, Kato T, Park EY. Int J Mol Sci, 2019, 20: 4228

56 Xu J, Dong Q, Yu Y, Niu B, Ji D, Li M, Huang Y, Chen X, Tan A. Proc Natl Acad Sci USA, 2018, 115: 8757-8762

57 Yarger JL, Cherry BR, van der Vaart A. Nat Rev Mater, 2018, 3: 18008

58 Whittall DR, Baker KV, Breitling R, Takano E. Trends Biotechnol, 2021, 39: 560-573

59 Flemming HC, Wingender J. Nat Rev Microbiol, 2010, 8: 623-633

60 Fowler DM, Koulov AV, Balch WE, Kelly JW. Trends Biochem Sci, 2007, 32: 217-224

61 Hudalla GA, Sun T, Gasiorowski JZ, Han H, Tian YF, Chong AS, Collier JH. Nat Mater, 2014, 13: 829-836

62 Fagan RP, Fairweather NF. Nat Rev Microbiol, 2014, 12: 211-222

63 Sleytr UB, Schuster B, Egelseer EM, Pum D. FEMS Microbiol Rev, 2014, 38: 823-864

64 Ilk N, Egelseer EM, Sleytr UB. Curr Opin Biotechnol, 2011, 22: 824831

65 Park DM, Reed DW, Yung MC, Eslamimanesh A, Lencka MM, Anderko A, Fujita Y, Riman RE, Navrotsky A, Jiao Y. Environ Sci Technol, 2016, 50: 2735-2742

66 Moll D, Huber C, Schlegel B, Pum D, Sleytr UB, Sára M. Proc Natl Acad Sci USA, 2002, 99: 14646-14651

67 Manea F, Garda VG, Rad B, Ajo-Franklin CM. Biotechnol Bioeng, 2020, 117: 912-923

68 Ben-Sasson AJ, Watson JL, Sheffler W, Johnson MC, Bittleston A, Somasundaram L, Decarreau J, Jiao F, Chen J, Mela I, Drabek AA, Jarrett SM, Blacklow SC, Kaminski CF, Hura GL, De Yoreo JJ, Kollman JM, Ruohola-Baker H, Derivery E, Baker D. Nature, 2021, 589: 468-473

69 Cooper A, Kennedy MW. Biophysl Chem, 2010, 151: 96-104

70 Mackenzie CD, Smith BO, Meister A, Blume A, Zhao X, Lu JR, Kennedy MW, Cooper A. Biophysl J, 2009, 96: 4984-4992
71 Park WM, Champion JA. J Am Chem Soc, 2014, 136: 17906-17909

72 Hu J, Wang G, Liu X, Gao W. Adv Mater, 2015, 27: 7320-7324

73 Wang Z, Guo J, Liu X, Sun J, Gao W. J Control Release, 2020, 328 : 444-453

74 Choi JM, Holehouse AS, Pappu RV. Annu Rev Biophys, 2020, 49: 107-133

75 Zeng M, Shang Y, Araki Y, Guo T, Huganir RL, Zhang M. Cell, 2016, 166: 1163-1175.e12

76 Roberts S, Dzuricky M, Chilkoti A. FEBS Lett, 2015, 589: 2477-2486

77 McDaniel JR, Radford DC, Chilkoti A. Biomacromolecules, 2013, 14 : 2866-2872

78 Simon JR, Carroll NJ, Rubinstein M, Chilkoti A, López GP. Nat Chem, 2017, 9: 509-515

79 Roberts S, Miao V, Costa S, Simon J, Kelly G, Shah T, Zauscher S, Chilkoti A. Nat Commun, 2020, 11: 1342

80 Dzuricky M, Rogers BA, Shahid A, Cremer PS, Chilkoti A. Nat Chem, 2020, 12: 814-825

81 Merg AD, Touponse G, Genderen E, Blum TB, Zuo X, Bazrafshan A, Siaw HMH, McCanna A, Brian Dyer R, Salaita K, Abrahams JP, Conticello VP. J Am Chem Soc, 2020, 142: 19956-19968

82 Murphy WL, Dillmore WS, Modica J, Mrksich M. Angew Chem Int Ed, 2007, 46: 3066-3069

83 Wu J, Li P, Dong C, Jiang H, Bin Xue H, Gao X, Qin M, Wang W, Bin Chen W, Cao Y. Nat Commun, 2018, 9: 620-631

84 Petka WA, Harden JL, McGrath KP, Wirtz D, Tirrell DA. Science, 1998, 281: 389-392

85 Sun F, Zhang WB, Mahdavi A, Arnold FH, Tirrell DA. Proc Natl Acad Sci USA, 2014, 111: 11269-11274

86 Wang R, Yang Z, Luo J, Hsing IM, Sun F. Proc Natl Acad Sci USA, 2017, 114: 5912-5917

87 Kou S, Yang Z, Sun F. ACS Appl Mater Interfaces, 2017, 9: 20352039

88 Kou S, Yang Z, Luo J, Sun F. Polym Chem, 2017, 8: 6158-6164

89 Yang Z, Yang Y, Wang M, Wang T, Fok HKF, Jiang B, Xiao W, Kou S, Guo Y, Yan Y, Deng X, Zhang WB, Sun F. Matter, 2020, 2: 233249

90 Wang XW, Liu D, Yin GZ, Zhang W-B. Tuning mechanical properties of protein hydrogels: Inspirations from nature and lessons from synthetic polymers. In: Yang G, Xiao L, Lamboni L, Eds. Bioinspired Materials Science and Engineering. Hoboken: John Wiley \& Sons, Inc.

91 Gerber LC, Koehler FM, Grass RN, Stark WJ. Proc Natl Acad Sci USA, 2012, 109: 90-94

92 Gerber LC, Koehler FM, Grass RN, Stark WJ. Angew Chem Int Ed, 2012, 51: 11293-11296

93 Chen AY, Deng Z, Billings AN, Seker UOS, Lu MY, Citorik RJ, Zakeri B, Lu TK. Nat Mater, 2014, 13: 515-523

94 Liu X, Tang TC, Tham E, Yuk H, Lin S, Lu TK, Zhao X. Proc Natl Acad Sci USA, 2017, 114: 2200-2205

95 Wang Y, An B, Xue B, Pu J, Zhang X, Huang Y, Yu Y, Cao Y, Zhong C. Nat Chem Biol, 2021, 17: 351-359

96 Huang J, Liu S, Zhang C, Wang X, Pu J, Ba F, Xue S, Ye H, Zhao T, Li K, Wang Y, Zhang J, Wang L, Fan C, Lu TK, Zhong C. Nat Chem Biol, 2019, 15: 34-41 